Recibido: 13/05/2021 --- Aceptado: 23/06/2021 --- Publicado: 19/07/2021

\title{
LA POLARIZACIÓN Y ECHO CHAMBERS EN EL DEBATE DE LA \#LEYDEEUTANASIA EN TWITTER
}

\section{POLARIZATION AND ECHO CHAMBERS IN \#LEYDEEUTANASIA DEBATE IN TWITTER}

(10) (8) Eider Urcola Eceiza': Universidad del País Vasco/ Euskal Herriko Unibertsitatea.

eider.urcola@ehu.eus

Imanol Elezgarai Ibañez: Universidad del País Vasco/ Euskal Herriko Unibertsitatea.

imanol.elezgaray@ehu.eus

\section{Cómo citar el artículo:}

Urcola Eceiza, E. y Elezgarai Ibañez, I. (2021). La polarización y echo chambers en el debate de la \#LeydeEutanasia en Twitter. Revista de Comunicación de la SEECI, 54, 187-204. http://doi.org/10.15198/seeci.2021.54.e723

Agradecemos el apoyo recibido por las instituciones del Gobierno Vasco/Eusko Jaurlaritza y Universidad del País Vasco/Euskal Herriko Unibertsitatea para la realización de los proyectos de tesis doctorales en los que se enmarca este trabajo. La investigación inició el 15 de octubre del 2020 y finalizó el 24 de febrero del 2021.

\section{RESUMEN}

El diálogo político llevado a los extremos y el posicionamiento de los actores políticos en los márgenes del debate es una de las principales preocupaciones de los ciudadanos en España (CIS, 2020). Lejos de ser un fenómeno exclusivo de la clase política, este distanciamiento tiene su reflejo entre la propia ciudadanía que, impulsada por las características de las redes sociales, ha desarrollado una especial tendencia a escucharse a sí misma, y a evitar conectar con opiniones divergentes (Sunstein, 2003). El objetivo principal del presente estudio es analizar la polarización en Twitter en torno al debate de la \#LeydeEutanasia en España. Para el análisis de este fenómeno se ha utilizado el software NodeXL para la recogida de datos y Gephi para su visualización. Además, se ha recreado una tabla de encuadre emocional (Menseres, Del Campo y

\footnotetext{
${ }^{1}$ Eider Urcola Eceiza: Doctoranda en el departamento de Comunicación Audiovisual y Publicidad en la Universidad del País Vasco/Euskal Herriko Unibertsitatea. Realiza el doctorado con una beca en el marco del Programa Predoctoral de Formación de Personal Investigador.

eider.urcola@ehu.eus
} 
Rueda-Zárate, 2018) para poder analizar los tuits de los actores políticos españoles y profundizar en su contenido. De esta manera, la investigación ha demostrado la presencia de dos echo chambers o grupos polarizados en torno al debate, que se ajustan a los bloques ideológicos en el eje izquierda/derecha, y que también se observan en el lenguaje utilizado para exponer su postura, en el que sin embargo no se utiliza de forma mayoritaria un lenguaje ofensivo en las interacciones con el adversario ideológico. Estos datos reafirman los resultados de trabajos previos en este ámbito de estudio.

PALABRAS CLAVE: Twitter - Redes sociales - Análisis de clúster - Polarización Echo chambers - \#leydeeutanasia - bloques ideológicos.

\section{ABSTRACT}

Political dialogue taken to extremes and the positioning of political actors on the margins of the debate is one of the main concerns of citizens in Spain (CIS, 2020). Far from being a phenomenon exclusive to the political class, this distancing is reflected among citizens themselves who, driven by the characteristics of social networks, have developed a special tendency to listen to themselves and avoid connecting with divergent opinions (Sunstein, 2003). The main objective of this study is to analyse the polarization on Twitter around the \#LeydeEutanasia debate in Spain. For the analysis of this phenomenon, NodeXL software has been used for data collection and Gephi for its visualization. In addition, an emotional framing table (Menseres, Del Campo \& Rueda-Zárate, 2018) has been recreated in order to analyse the Tweets of Spanish political actors and study their content. In this way, the research has demonstrated the presence of two echo chambers or polarized groups around the debate, which are in line with the ideological blocks on the left/right axis, and which are also observed in the language used to expose their position. However, offensive language is not used in the majority of interactions with the ideological adversary. These data reaffirm the results of previous work in this field of study.

KEY WORDS: Twitter - Social media - Cluster analysis - Polarization - Echo chambers - \#leydeeutanasia - Ideological blocks

\section{A POLARIZAÇAO E ECHO CHAMBERS NO DEBATE DA\#LEYDEEUTANASIA NO TWITTER}

\section{RESUMO}

O dialogo politico levado aos extremos e o posicionamento dos atores politicos nas margens do debate é uma das principais preocupações dos cidadãos na espanha (CIS,2020). Longe de ser um fenômeno da classe política, este distanciamento, tem seu reflexo entre a própria cidadania que, impulsionada pelas características das redes sociais, tem desenvolvido uma especial tendência de se escutar a si mesma, e a evitar conectar com opiniões divergentes. (Sunstein, 2003). O objetivo principal do presente estudo é analisar a polarizaçao no twitter em torno ao debate da \#LeydeEutanasia na Espanha.Para a análise deste fenômeno se utilizou o software NEdXL para a coleta de dados e Gephi para sua visualização. Além disso foi retirado uma tabela de 
enquadramento emocional (Menores,Del campo y Rueda Zarate,2018) para poder analisar os tuits dos atores políticos espanhóis e aprofundar no seu conteúdo. Desta forma, a pesquisa tem demonstrado a presença de eco chambers ou grupos polarizados em torno do debate, que se ajustam aos blocos ideológicos no eixo esquerda/direita, e que também se observam na linguagem utilizada para expor sua postura, na que em sua maioria não se usa linguagem ofensiva nas interações com o adversário ideológico. Estes dados reafirmam os resultados prévios neste âmbito e estudo.

PALAVRAS CHAVE: Twitter - Redes sociais - Análise de cluster - Polarização - Echo chambers - \#leydeeutanasia - bloques ideológicos.

\section{INTRODUCCIÓN}

Una de las bases de la democracia es la libertad de la opinión pública, y para que la sociedad pueda opinar libremente necesita información libre y verdadera (UNESCO, -). Hasta hace unos años el pilar para esa libertad era la prensa escrita, televisión y radio. Hoy en día en cambio, la forma en que nos informamos, debatimos y formamos nuestras opiniones ha cambiado profundamente con la llegada de los medios de comunicación en línea y especialmente, las redes sociales (Törnberg, 2018: 1).

Las redes sociales, por su estructura, tienen el potencial para acercar a los ciudadanos a la esfera política y a su influencia (Castells, 2011) por cuanto promueven una participación ciudadana más activa en los asuntos políticos y en la creación y difusión de los contenidos (Rodríguez et al., 2010; Matakos et al., 2017). Las redes, además, aumentan la cantidad de información que tenemos a nuestra disposición y la variedad de las opciones a elegir (Sunstein, 2003: 65). Reuniendo en un mismo espacio a individuos de diferentes países y culturas que pueden expandir sus horizontes con nuevos puntos de vista (Papacharissi, 2002:23).

Sin embargo, incluso aquellas herramientas que planteaban la oportunidad de democratizar las relaciones sociales y unir en la discusión a personas que sin ellas difícilmente entrarían en contacto, han mostrado zonas de debilidad a la hora de cumplir con las expectativas creadas (Bakshy et al., 2015: 1130; Vaccari et al., 2016: 2; Törnberg, 2018:1; Zuiderveen Borgesius et al.,2016).

Las redes también pueden ser utilizadas con otras intenciones, fomentando la crispación, la división y la fractura social, utilizando para ello hechos no contrastados, o afirmaciones subjetivas que no buscan más que la simple descalificación de las ideas del adversario político, o la manipulación (Rojas, 2007). Y es que, más que en una deliberación racional y pausada, las redes sociales funcionan modulando y distribuyendo una atmósfera, un sentimiento público, que a su vez condiciona a los usuarios, imponiendo a menudo las condiciones mayoritarias que circulan por la red (Maldonado, 2016).

\subsection{Polarización}

Revista de Comunicación de la SEECI. 2021, n 54, 187-204 
Al estar los usuarios mayoritariamente relacionados con opiniones afines, los polos opuestos de la opinión pública se alejan todavía más: se polariza la red (Lee et al., 2014:707). Lejos de un punto central de consenso (Baumann et al., 2020). Según Sunstein (2003:69): <<Inmersos en un continuo debate entre usuarios que comparten una similar línea de pensamiento, los individuos tienden a adoptar una posición más extremista que acentúa la que originariamente poseían >>.

Una red se considera polarizada si los usuarios pueden dividirse en al menos dos subgrupos altamente cohesionados que tendrán, posiblemente dos puntos de vista antagónicos (Guerra et al., 2013:215). Un espacio digital en el que los usuarios tienden a promover sus narraciones favoritas, a formar grupos concentrados (Bessi et al., 2016:2048) y a resistirse a la información que no se ajusta a sus creencias (Törnberg, 2018: 2; Quattburociocchi et al., 2016). Esto es un riesgo potencial para la democracia, ya que, en general, las personas que tienden a filtrar las opiniones opuestas son las que, precisamente, más necesitan oír otras opiniones (Sunstein, 2003: 70; Taber y Lodge, 2006:765).

Vaccari et al. (2016:1) han demostrado que los usuarios de twitter tienen más probabilidades de emplear los medios sociales para participar en las redes que apoyaban en lugar de desafiar los puntos de vista del usuario. Y a su vez la polarización de grupos aumentará de forma significativa si los individuos se consideran a sí mismos como parte de un grupo con una identidad compartida y un cierto grado de solidaridad. Si esta es la idea que tienen de sí mismos, la polarización de grupos es más probable y tenderá a agudizarse (Sunstein, 2003: 73).

Aun así, según la investigación de Barberá et al. (2015:1539), esta polarización y la segregación varía dependiendo de la naturaleza de los acontecimientos. A su vez, también observaron que el nivel de participación con otros usuarios de diferentes ideologías cambia según la opinión ideológica del usuario y conversación en la red.

\subsection{Echo Chambers}

En las últimas investigaciones ha ganado interés el carácter selectivo de la red enunciado por Sunstein (2003:70) - las llamadas echo chambers (Vaccari et al., 2016: 2; Sinderman et al., 2020: 1; Buder et al., 2021; 2). La investigación ha revelado que hay un vínculo entre las cámaras de eco y la polarización (Tonberg, 2018: 2; Sunstein, 2016:14; Stroud, 2010; Garrett, 2009: 280).

Las echo-chambers son un conjunto de nodos estrechamente conectados e inclinados a compartir una opinión común sobre una narrativa determinada (Törnberg, 2018: 2). Internet, y especialmente las redes sociales, funcionan a menudo como cámaras de eco en donde los individuos se exponen a opiniones generalmente afines a las suyas y pocas veces discrepan entre los interlocutores en la discusión (Vaccari et al., 2016: 2; Bakshy et al., 2015:1130). Existe por tanto una tendencia natural en la acción en las redes sociales, tanto humanas como digitales, que lleva a los individuos a no realizar elecciones, en lo que respecta al ocio y las noticias que desafíen su visión preexistente del mundo (Sunstein, 2003: 62). 
También se ha visto que los factores de personalidad, edad, género e ideología influyen a la hora de ser afectados por las cámaras de eco (Sinderman et al., 2020). A estos elementos puede añadírseles también el del anonimato, ya que estudios previos (Davidson et al., 2020: 7) han descubierto niveles de polarización particularmente altos cuando los miembros del grupo se presentan de forma relativamente anónima y cuando se da importancia a la identidad de grupo. A partir de ello es razonable plantearse que la polarización tiene más posibilidades de aparecer y de ser extrema cuando el hecho de ser miembro del grupo es algo importante y los individuos cuentan con el anonimato (Sunstein, 2003: 74).

Además, los investigadores Tsai, Tao, Chuan y Hong (2020) tras una investigación sobre retuits y menciones, concluyen que, aunque se pueden realizar menciones y retuits a usuarios que no son de la misma ideología, la mayoría de las interacciones (menciones y retuits) se realizan entre los miembros del grupo interno (Tsai et al., 2020:8).

Siguiendo con las interacciones, Buder et al. han demostrado que el sentimiento del mensaje, es decir, el lenguaje utilizado por los usuarios puede ser el precursor de la polarización, y en última instancia a la fractura de la sociedad (Buder et al., 2021:7).

\subsection{Polarización y echo chambers en la \#LeydeEutanasia}

Este clima cambiante en las redes sociales se ha convertido rápidamente en una amenaza, y se considera ahora un riesgo importante para la sociedad (Törnberg, 2018: 2; Matakos et al., 2017:1841). La Red está sirviendo a muchos para propagar el extremismo, precisamente porque la exposición frecuente de las personas a una posición extrema, junto con el indicio de que muchos individuos apoyan dicha posición, afectará a esas personas expuestas y predispuestas a creer en ella. Como consecuencia se obtendrá un alto grado de fragmentación, ya que diferentes personas, que, en un principio no tenían unas opiniones demasiado concretas y quizá no muy diferentes, acaban en posiciones completamente opuestas como resultado de lo que leen y ven (Sunstein, 2003: 74).

\section{OBJETIVOS}

El objetivo de este estudio es analizar la polarización en Twitter. Para poder realizar la investigación se ha tomado como objeto de estudio el debate sobre la Ley de Eutanasia de España votada en el congreso de los diputados el 17 de diciembre del 2020. No es la primera vez que el congreso vota una propuesta de legalización de la Eutanasia, de hecho, se votó y se rechazó hasta cuatro veces antes de la aprobación en esta última ocasión (Bueno y Martínez, 2019). La última hace tan solo tres años, en 2018, presentada entonces por Unidas Podemos, en una sesión en la que también se rechazó una propuesta de ley de muerte digna de Ciudadanos. La propuesta de la ley, trabajada durante meses generó también una importante polémica tanto en los medios de comunicación tradicionales como entre los propios partidos políticos (Hermida, 2020), de forma que como era de esperar, la discusión en torno a la 
aprobación de la ley la cámara baja tuvo también un gran alcance en la red social Twitter como demuestran las decenas de miles de tuits escritos en torno a la temática de la Ley de Eutanasia algunos de los cuales se analiza en estas páginas.

\section{METODOLOGÍA}

El material consta de tuits recogidos el día 17 de diciembre de 2020 con el hashtag \#LeydeEutanasia mediante la plataforma NodeXL. Se trata de un software que recaba los últimos tuits en orden estrictamente cronológico (los últimos tuits escritos en la red social) relacionados con el hashtag demandado (Kuz et al., 2015: 206). Ha sido un programa ampliamente utilizado en los últimos años para la recolección del dato en redes sociales para su posterior análisis (Rim et al., 2020; Weber et al., 2013; Wu, 2013). En total se han recogido 22.986, la cifra máxima que permite recoger el software en su versión para la academia, que permite realizar un muestreo adecuado para analizar a través de este el resto de la red que participa en este debate.

Dichos tuits se han visualizado en el software Gephi.9.2, qué sirve por un lado para realizar el cálculo de estadísticas relacionadas con la red como pueden ser la densidad del grafo, el diámetro del mismo o el grado de entrada y salida de los usuarios (McSweeney, 2009), y por otro lado, permite, a través de diversos algoritmos, el cálculo de coeficientes de clusterización (Cherven, 2015), que permite visualizar los diversos grupos implicados en el debate, clasificados en base a la interrelación entre los mismos, tanto la previa al debate (quien sigue a quien y viceversa) como la resultante en el propio debate (las interacciones que tienen entre ellos unos y otros). Este software ha sido utilizado en varias investigaciones de la misma temática (Lumban Gaol et al.,2020; Gualda et al., 2015). En concreto se ha utilizado el algoritmo OpenOrd (ibíd., 2015: 35) que ayuda a generar grafos de red muy rápidamente, y es más adecuado para redes muy grandes debido a la pérdida de precisión en aras de una mayor velocidad.

De estos tuits se han elegido aleatoriamente mediante el programa estadístico SPSS 5691 tuits (una muestra del $\% 25$ de los datos) para su posterior análisis de contenido. Estos tuits han sido analizados manualmente para determinar la perspectiva de los usuarios dentro de este debate. Para ello se ha utilizado la clasificación de encuadre emocional de Meneses et al. (2018: 46). En base a esta tabla hemos formulado las siguientes categorías para su mejor adaptación al objetivo del estudio: apoyo (mantiene claramente una postura favorable), repudio/desagrado (explícita su rechazo a la norma), ofensivo negativo (contrario a la aprobación de la ley pero para ello utiliza términos ofensivos para la persona objetivo del mensaje), ofensivo positivo (a favor de la aprobación pero a la hora de sostener su opinión utiliza un lenguaje ofensivo para otros usuarios), tuit informativo (se limita a informar sobre algún aspecto relativo a la ley pero sin entrar a valorarla), no-alineados (aquellos usuarios que no muestran con claridad una opinión contraria o favorable a la ley) y otros (para esos tuits que no encajan en ninguna otra categoría).

\section{ANÁLISIS Y RESULTADOS}




\subsection{Análisis de grafo}

Se reflejan tres grupos principales en el análisis: el primer grupo -en la figura 1 en color rojo/ magenta- situado a la izquierda ideológica incluye a las diversas sensibilidades y fuerzas políticas de izquierdas, colectivos y medios de comunicación. En total este grupo representan un $32,35 \%$ de los nodos y el $35,13 \%$ de las aristas. Consideramos grupo a la suma de varios clústeres los cuales a su vez constituyen un conjunto de nodos.

En el segundo grupo -en la figura 1 en color verde- situado a la derecha del gráfico se sitúan los grupos de la derecha ideológica junto a otros colectivos y medios de comunicación, y miembros y asociaciones de la órbita de la Iglesia Católica. Agrupan al $33,16 \%$ de los nodos y el $30,93 \%$ de las aristas.

Y por último hay un tercer grupo en la parte inferior del grafo -en color gris-. En este grupo se mezclan dos clústeres. En el primero de ellos se sitúan periodistas, políticos y usuarios chilenos, que, debido a las circunstancias del país andino, donde a la vez que en España se estaba debatiendo la ley de Eutanasia en el congreso, aparecen también incluidos en el hashtag sobre la eutanasia. En el segundo clúster se recoge un grupo más que engloba a varias organizaciones y medios de comunicación tanto del ámbito de la izquierda como de la derecha. Al no ser posible cuantificar el peso de cada clúster dentro del grupo, se ha decidido no contabilizar este grupo en la clasificación de grupos de izquierda y derecha. El clúster chileno No 354, representa un total de $5,76 \%$ de los nodos y $4,30 \%$ de las aristas. Mientras que el otro grupo ( $\mathrm{n}^{\circ}$ 636) engloba a varias organizaciones y medios de comunicación tanto del ámbito de la izquierda como de la derecha. 
Imagen 1: Bloques ideológicos en el grafo.

Fuente: Elaboración propia.

El primer grupo denominado como el de la izquierda ideológica engloba a 5 de los principales clústeres. Por tamaño en cuanto al porcentaje de nodos de toda la red, de mayor a menor, son los clústeres número: 531 (8,98\% de los nodos totales), 2 (7,8\%), $1(7,04 \%)$ y $32(4,79 \%)$.

El segundo grupo es el que clasificamos como el que engloba a 6 clústeres relacionados con el espectro ideológico de la derecha: tomando en consideración el porcentaje de nodos de la red, de mayor a menor, engloba a los clústeres número: 372 (9,82\% del total de los nodos), 365 (9,58\%), 255 (5,13\%), $387(4,19 \%), 272$ $(4,12 \%), 485(4 \%)$ y el clúster $0(2,55 \%)$.

En lo que a usuarios dentro de estos clústeres se refiere se da la circunstancia de que los actores referenciales de los clústeres son usuarios en su mayoría que no ocupan un cargo público.

\subsubsection{Grado de entrada}

En este apartado se desglosan los usuarios en cuanto al grado de entrada, que registra el número de interpelaciones que recibe cada uno de los usuarios que 
conforman la red. Se incluye entre las interpelaciones al total de menciones, likes y retuits recibidos por el usuario.

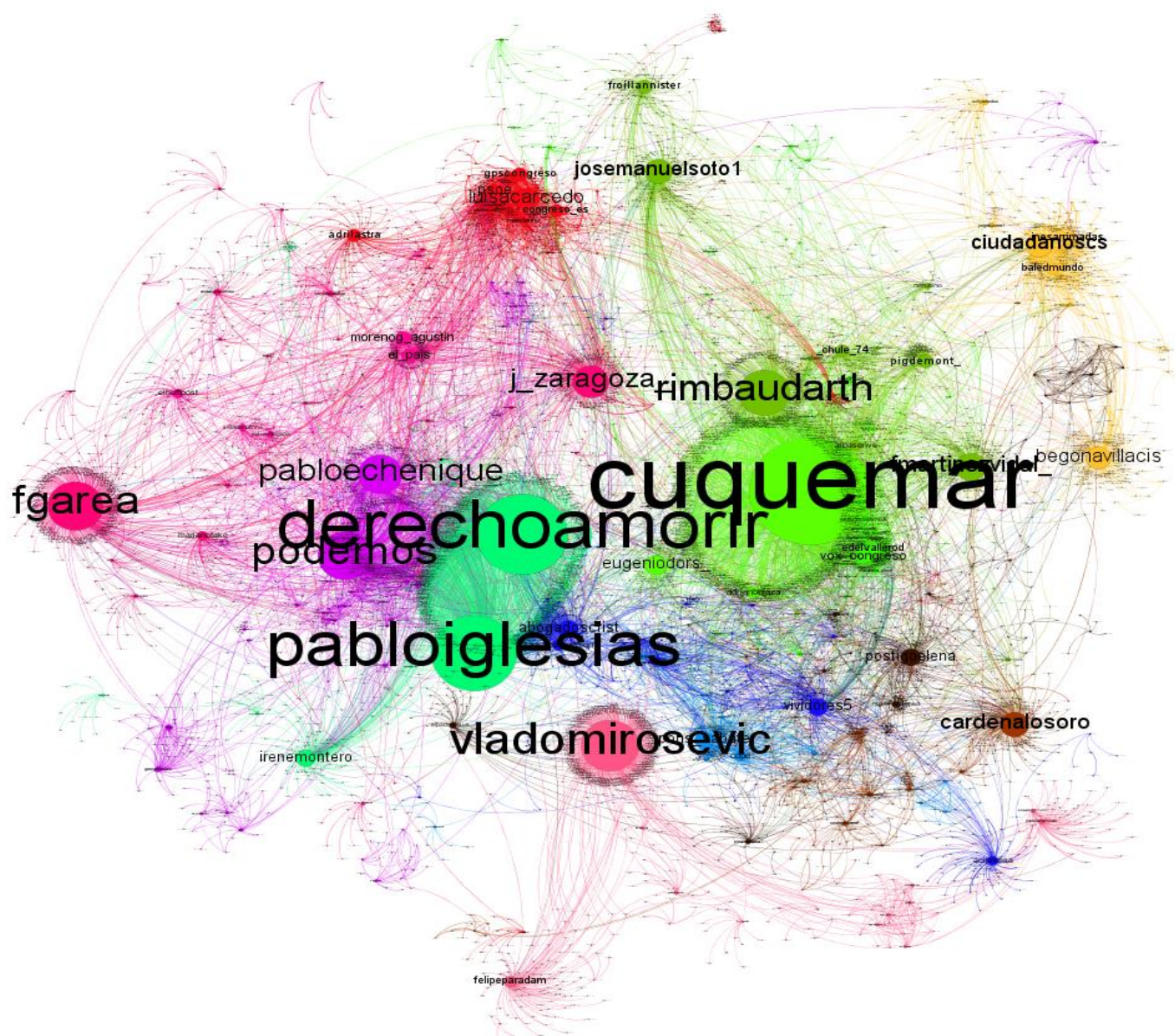

Imagen 2: Usuarios referentes en cada clúster.

Fuente: Elaboración propia.

En orden ascendente al descendente en cuanto al grado de entrada: el mayor representante de la red es Marcial Cuquerella (@cuquemar), que se define como empresario de medios y tecnología, tiene un grado de entrada de 1130 , y es miembro del clúster n0365. Junto a él, en su mismo clúster, se encuentran otros tuiteros como @adrianbelaza -grado de entrada de 113-, o Alberto Asensi (@albasenve) -grado de entrada de 96-.

El siguiente usuario más destacado es @derechoamorir, la plataforma Derecho a Morir Dignamente la asociación defensora de la ley, con un coeficiente de grado de entrada de 842. Le sigue el vicepresidente de asuntos sociales del Gobierno de España Pablo Iglesias (@PabloIglesias), con un coeficiente de 793. El cual se sitúa en la centralidad del debate, pero esto no significa que él tenga una postura centralista, sino que Pablo Iglesias es nodo referente entre los usuarios que componen la posición favorable a la aprobación de la ley. 
Les sigue, en el clúster n0531 Fernando Barea (@Fgarea), director adjunto del medio digital El Confidencial (grado de entrada de 508) y otras personas que por sus interacciones en el debate no aparecen en el gráfico directamente relacionados con ningún partido político en concreto en este debate. Por detrás, el clúster n०2, que corresponde al partido político Podemos (@PODEMOS) -grado de entrada de 497- y su portavoz parlamentario Pablo Echenique (@PabloEchenique) -grado de entrada de 402-. Este clúster está más relacionado con el partido político y sus agrupaciones.

En el clúster n0372 nos encontramos con el fenómeno inverso, los referentes principales son personas no directamente relacionadas con un partido político @rimbaudarth (grado de entrada 471) o @josemanuelsoto (grado de entrada 247), salvo el concejal de Vox de Madrid Fer Martínez Vidal (@fmartinesvidal_) con un grado de entrada de 276.

El siguiente representante más llamativo es el Arzobispo de Madrid Carlos Osoro (@cardenalosoro) -grado de entrada de 255-. Y junto a él se agrupan otros miembros de la diócesis como el secretario general de la conferencia episcopal Luis Arguello (@MonsArguello) -grado de entrada de 45-, o el arzobispo de Toledo Francisco Cerro (@Obispofcerro) -grado de entrada de 39-.

El usuario referencial en cuanto al grado de entrada en el cluster n०272 es el partido político Ciudadanos (@CiudadanosCs) -grado de entrada de 238-, y a su alrededor se sitúan Edmundo Bal (@BalEdmundo) -grado de entrada de 117- y Begoña Villacis (@begonavillacis) -grado de entrada de 224-.

En un nuevo clúster nos encontramos a Luisa Carcedo (@luisacarcedo) y varios miembros del Partido Socialista (@PSOE), este grupo situado en la parte superior incluye tanto el partido político (con un grado de entrada de 154) como el grupo parlamentario socialista y a varios de sus referentes como Adriana Lastra (@Adrilastra) - grado de entrada de 121-, u Odón Elorza (@odonelorza2011) - grado de entrada de 50-.

El grupo n0387 lo conforman usuarios anónimos como @eugeniodors, el usuario con el grado más alto del clúster -193-, personas de otros ámbitos como Elena Postigo (@PostigoElena) profesora de filosofía y bioética -grado de entrada de 174-, o @princesatesa, con un grado de entrada de 41.

El clúster n0485 lo engloba @Vox_congreso, la cuenta del grupo parlamentario de VOX que tiene un grado de entrada de 142, Emilio del Valle (@edelvallerod) con un grado de entrada de 122, Diputado de Vox en el Congreso, y junto a él se sitúan otros políticos y medios como Macarena Ollona (@Macarena_Olona) -grado de entrada de 48-, Jorge Buxade (@Jorgebuxade) - grado de entrada de 44- o el medio de comunicación La Gaceta (@gaceta_es) -grado de entrada de 48-.

En un último grupo n 00 se encuentran varios miembros del Partido Popular, con su presidente Pablo Casado (@pablocasado_) a la cabeza -grado de entrada de 18-, aunque en este grupo destaca también el presidente del Gobierno de España, Pedro 
Sánchez (@sanchezcastejon). Esto es debido a que los miembros del partido popular, contrarios en lo político a Sánchez, le interpelan reiteradamente en el debate, como se puede observar en su alto valor de grado de entrada -107-.

Tabla 1. Grado de entrada

\begin{tabular}{|c|c|c|c|}
\hline \multicolumn{5}{|c|}{ Usuarios referentes de clúster según su grado de entrada } \\
\hline Número de Clúster & Porcentaje de nodos & Usuarios referentes & Grado de entrada \\
\hline 365 & $9,58 \%$ & @cuquemar & 1130 \\
\hline 1 & $7,04 \%$ & @derechoamorir & 842 \\
\hline 531 & $8,98 \%$ & @FBarea & 508 \\
\hline 2 & $7,8 \%$ & @PODEMOS & 497 \\
\hline 372 & $9,82 \%$ & @rimbaudarth & 471 \\
\hline 255 & $5,13 \%$ & @cardenalosoro & 255 \\
\hline 272 & $4,12 \%$ & @CiudadanosCs & 238 \\
\hline 32 & $4,79 \%$ & @Luisacarcedo & 219 \\
\hline 387 & $4,19 \%$ & @eugeniodors & 193 \\
\hline 485 & $4 \%$ & @Vox_congreso & 142 \\
\hline 0 & $2,55 \%$ & @sanchezcastejon & 107 \\
\hline
\end{tabular}

Fuente: Elaboración propia

\subsubsection{Grado de salida}

Denominamos grado de salida a las interacciones realizadas por los usuarios de la red para con el resto de usuarios, entendiéndose por interacciones las menciones, retuits y likes realizadas al resto de usuarios. Debemos puntualizar que, en lo referente al grado de salida, la diferencia en la cantidad de los usuarios destacados de cada grupo y el resto de usuarios del grupo es bastante mayor que en el grado de entrada. El grafo muestra de manera muy visual que tan solo unos pocos usuarios de cada grupo participan muy activamente (realizan múltiples interpelaciones hacia otros usuarios) en el debate. Cada uno de los clústeres tiene uno o dos usuarios cuya actividad -representada visualmente por su tamaño en el grafo- es exponencialmente superior al resto destacando en lo visual de manera clara en el grafo. A su vez, cabe señalar que la clasificación de los clústeres no varía en relación a su tamaño en la red general, por lo que en este apartado del grado de salida se identificarán únicamente los usuarios de mayor grado de salida, sin destacar el posicionamiento del clúster.

De mayor a menor en relación al grado de salida de los usuarios, destaca Podemos Alicante Oeste, del clúster no 2 (@p_alicanteoeste), con 37. Por detrás de este, @1denmadrid (clúster no 1) -grado de salida 23-, @prensacee del clúster no 255 con 22, @pilargonzalezl1 del clúster no 365, @meme_montero del clúster no 32 y con grado de salida 17. Le sigue de cerca con 17 @joselisa17 del clúster no 372 . Los siguientes en la lista son @martinaguirrecm con 14, @pinkiewarriorpw del clúster no 272 tiene el siguiente grado de salida más alto -13-, seguido por @juanbasangulo del n $0-12-$, @jgdeaguilar del n 387 con 11 y por último @mfedorral del nº 531 con 8. 
Tabla 2. Grado de salida

\begin{tabular}{|c|c|c|c|}
\hline \multicolumn{5}{|c|}{ Usuarios de referencia de clúster según su grado de salida } \\
\hline Número de Clúster & Porcentaje de nodos & Usuarios referentes & Grado de salida \\
\hline 2 & $7,8 \%$ & @p_alicanteoeste & 37 \\
\hline 1 & $7,04 \%$ & @1denmadrid & 23 \\
\hline 255 & $5,13 \%$ & @prensacee & 22 \\
\hline 365 & $9,58 \%$ & @pilargonzalez1 & 20 \\
\hline 32 & $4,79 \%$ & @meme_montero & 17 \\
\hline 372 & $9,82 \%$ & @joselisa17 & 17 \\
\hline 485 & $4 \%$ & @martinaguirrecm & 14 \\
\hline 272 & $4,12 \%$ & @pinkiewarrior & 13 \\
\hline 0 & $2,55 \%$ & @juanbasangulo & 12 \\
\hline 387 & $4,19 \%$ & @jdeaguilar & 11 \\
\hline 531 & $8,98 \%$ & @mfedorral & 8 \\
\hline
\end{tabular}

Fuente: Elaboración propia

\subsection{Clasificación de encuadre emocional}

El análisis del lenguaje emocional ha revelado que tal como muestra la imagen del análisis de Gephi, los tuits están divididos también por el lenguaje: 2394 tuits (42,06\%) muestran apoyo a la ley y 1993 tuits $(35,00 \%)$ en contra.

Además de los dos grupos principales, destaca la cifra de 242 tuits de ofensa negativa, es decir, que la polarización es alta pero el lenguaje utilizado no es especialmente ofensivo. También relevante que 724 tuits analizados son informativos, esto es, hay un espacio para la información más objetiva donde se explican aspectos relacionados con la ley y otras convocatorias para actividades, como, por ejemplo, convocatorias a manifestaciones o similares.

Otros datos menos relevantes son las ofensas positivas 102 tuits $(1,79 \%)$, los noalineados 8 tuits $(0,14 \%)$, y por último otros 229 tuits $(4,02 \%)$.

Tabla 3. Encuadre emocional

\begin{tabular}{|c|c|c|}
\hline Categoría & Cantidad de Tuits & Porcentajes \\
\hline Apoyo & 2394 & $42,06 \%$ \\
\hline Repudio/Desagrado & 1992 & $35,00 \%$ \\
\hline Ofensivo negativo & 242 & $4,25 \%$ \\
\hline Ofensivo positivo & 102 & $1,79 \%$ \\
\hline Informativo & 724 & $12,72 \%$ \\
\hline No alineados & 8 & $0,14 \%$ \\
\hline Otros & 229 & $4,02 \%$ \\
\hline Total & 5691 & $100 \%$ \\
\hline
\end{tabular}

Fuente: Elaboración propia 


\section{DISCUSIÓN Y CONCLUSIONES}

El análisis de los resultados ofrece un mapa visual fácilmente identificable en el que se simplifica un debate enormemente complejo, y en el que interactúan una gran cantidad de factores, actores y variables. Valiéndose de varias técnicas se pudo identificar a los sujetos más relevantes dentro del objeto de estudio, valiéndose de técnicas cuantitativas, y su posterior análisis de contenido a través de una técnica cualitativa, que permitió la secuenciación de los tuits y la orientación opinativa, a favor o en contra, de los mismos respecto al debate analizado.

Varias de las hipótesis formuladas al inicio del estudio se ven confirmadas tanto por el grafo relacional de los clústeres, como por el posterior análisis de contenido y su traslación al encuadre emocional. Se valida, por un lado, la hipótesis de que los debates entre los diferentes partidos políticos en torno a la aprobación de la ley de eutanasia, fuera de las redes sociales, tiene traslación directa a la red social Twitter. Y que esta discusión no se da únicamente entre los propios partidos o sus representantes, sino que se extiende a otros usuarios, así como a colectivos sociales o medios de comunicación. Además, estos resultados sobre la división ideológica/partidista de clúster en Twitter también van en acuerdo con las conclusiones de Conover et al. (2011:95)

Podemos afirmar también que la red refleja con claridad la presencia de al menos dos grupos de clústeres diferenciados entre sí en cuanto a su opinión respecto del debate sobre la ley de eutanasia, dos echo chambers destacadas, que si bien tienen una estrecha relación entre ellas, y un interrelación directa en forma de aristas que unen a los nodos unos con otros, no tienen un contacto tan estrecho con el grupo de opinión adversario, creando un vacío comunicativo entre ellas, dando lugar a una evidente polarización entre las opiniones favorables y contrarias a la aprobación de la ley. Los resultados concuerdan con los estudios previos realizados por: Garimella y Weber (2017), Vaccari et al. (2016), Quattrociocchi et al. (2016), Colleoni et al. (2014), Hahn et al. (2015) entre otros.

Además, esto se apoya en la idea principal de Barberá et al. (2015), donde remarcaban que la naturaleza del evento cambiaría el curso de la conversación y polarización, y que esto tendría matices ideológicos. Además del propio evento, el fenómeno de la polarización varía también país a país y dependiendo de las circunstancias y condicionantes políticas de cada uno de ellos (Urman, 2020). Esta polarización se da además en una correlación de fuerzas muy pareja en cuanto al porcentaje de tuits a favor y en contra, con un estrecho margen entre los usuarios que muestran su apoyo a la aprobación de la ley, el \%42 de los tuits lo es, frente a quienes se oponen a la misma: el $35 \%$.

Sin embargo, es de destacar que esa polarización estructural frente al debate, no se traslada en la misma medida al lenguaje utilizado por ambos grupos a la hora de interactuar entre ellos. El porcentaje de tuits favorables y contrarios se dan en un número mucho mayor que aquellos que se dirigen al adversario ideológico en términos ofensivos: tan solo un $4,25 \%$ de los tuits realizados por usuarios contrarios a la 
aprobación de la ley se escriben en términos ofensivos, mientras que aquellos tuits ofensivos escritos a favor de la aprobación de la ley son solo el 1,79\%. Estos resultados van en la línea de Buder et al. (2021:7), señalando que el lenguaje utilizado es precursor a la división de opiniones marcando la polarización.

El planteamiento metodológico diseñado para esta investigación ofrece una fotografía estática de una discusión que, sin embargo, tiene un desarrollo dinámico. Si bien el estudio del debate en el momento de la aprobación de la ley ofrece una información relevante acerca de la estructura del mismo, y los términos discursivos en que este se da, esto no es óbice para que dicha estructura o el marco discursivo no pueda evolucionar o variar en el tiempo. Son interesantes, por tanto, aquellos estudios que dieran continuidad en el tiempo al estudio, a fin de comparar la posición y las formas de los discursos de unos y otros y las motivaciones detrás de ese proceso de cambio, para tener una comprensión mayor y más completa del fenómeno a estudiar.

A su vez, la clasificación de los tuits en el encuadre emocional se ha realizado en base a una categorización favorable, contraria o neutral, sin entrar a valorar la motivación detrás de cada postura. Si bien a lo largo del análisis de contenido de los tuits se ha observado que en un amplio porcentaje los tuits a favor denotaban el deseo de una ampliación de los derechos sociales, y muchos de los emitidos en contra argumentan motivos religiosos o morales para oponerse a la aprobación de la ley, sería interesante incluir un análisis metodológico que permita clasificar las motivaciones detrás de las posturas de unos y otros. El desarrollo de estas cuestiones plantea como pertinentes nuevas investigaciones en torno a los fenómenos de polarización y las motivaciones que llevan a la misma.

\section{REFERENCIAS}

Arias-Maldonado, M. (2016). La democracia sentimental: política y emociones en el siglo XXI. Página indómita.

Bakshy, E., Messing, S., y Adamic, L. A. (2015). Political science. Exposure to ideologically diverse news and opinion on Facebook. Science, 348(6239), 11301132. https://10.1126/science.aaa 1160

Barberá, P., Jost, J. T., Nagler, J., Tucker, J. A., y Bonneau, R. (2015). Tweeting from left to right: Is online political communication more than an echo chamber? Psychological Science, 26(10), 1531-1542. https://doi.org/10.1177\%2F0956797615594620

Bessi, A., Petroni, F., Del Vicario, M., Zollo, F., Anagnostopoulos, A., Scala, A., Caldarelli, G. y Quattrociocchi, W. (2016). Homophily and polarization in the age of misinformation. The European Physical Journal Special Topics, 225(10), 2047-2059. https://doi.org/10.1140/epjst/e2015-50319-0 
Urcola Eceiza, E. y Elezgarai Ibañez, I. La polarización y echo chambers en el debate de la \#LeydeEutanasia en Twitter

Buder, J., Rabl, L., Feiks, M., Badermann, M. y Zurstiege, G. (2021). Does negatively toned language use on social media lead to attitude polarization? Computers in Human Behavior, 116, 106663. https://doi.org/10.1016/j.chb.2020.106663

Bueno, A. y Martínez, H. L. (2019). Todas las veces que la eutanasia llegó al Congreso. El País. https://bit.ly/3k219Iz

Castells, M. (2011). The rise of the network society. John wiley \& sons.

Centro de Investigaciones Sociológicas (Ed.) (2020). Barómetro de diciembre 2020. https://bit.ly/3dGCXYw

Cherven, K. (2015). Mastering Gephi network visualization. Packt Publishing Ltd.

Colleoni, E., Rozza, A. y Arvidsson, A. (2014). Echo chamber or public sphere? Predicting political orientation and measuring political homophily in Twitter using big data. Journal of Communication, 64(2), 317-332. https://doi.org/10.1111/jcom.12084

Conover, M., Ratkiewicz, J., Francisco, M., Gonçalves, B., Menczer, F. y Flammini, A. (2011). Political polarization on twitter. Paper presented at the Proceedings of the International AAAI Conference on Web and Social Media, 5(1). https://bit.ly/2NWsgq5

Davidson, S. S., Bradshaw Hoppock, A., Rohmeyer, R. A., Keebler, J. y Frederick, C. M. (2020). Deindividuation in Anonymous Social Media: Does Anonymous Social Media Lead to an Increase in Non-Normative Behavior? https://bit.ly/3knzgZ1

Gaol, F. L., Maulana, A. y Matsuo, T. (2020). News consumption patterns on Twitter: fragmentation study on the online news media network. Heliyon, 6(10). https://doi.org/10.1016/j.heliyon.2020.e05169

Garimella, V. R. K. y Weber, I. (2017). A long-term analysis of polarization on Twitter. Paper presented at the Proceedings of the International AAAI Conference on Web and Social Media, 11(1). https://bit.ly/3pY1gUx

Garrett, R. K. (2009). Echo chambers online?: Politically motivated selective exposure among Internet news users. Journal of Computer-Mediated Communication, 14(2), 265-285. https://doi.org/10.1111/j.1083-6101.2009.01440.x

Gualda, E. y Borrero, J. D. (2015). La'Spanish Revolution'en Twitter (2): Redes de hashtags (\#) y actores individuales y colectivos respecto a los desahucios en España. Redes. Revista Hispana Para El Análisis De Redes Sociales, 26(1), 1-22. https://bit.ly/3bsyAxm

Guerra, P., Meira, W. Jr., Cardie, C. y Kleinberg, R. (2013). A measure of polarization on social media networks based on community boundaries. Paper presented at the 
Urcola Eceiza, E. y Elezgarai Ibañez, I. La polarización y echo chambers en el debate de la \#LeydeEutanasia en Twitter

Proceedings of the International AAAI Conference on Web and Social Media, 7(1). https://bit.ly/2NxYPdS

Hermida, X. (2020, december 19). El Congreso aprueba la primera ley de eutanasia con amplia mayoría. El País. https://bit.ly/3k1030j

Hahn, K. S., Ryu, S. y Park, S. (2015). Fragmentation in the Twitter following of news outlets: The representation of South Korean users' ideological and generational cleavage. Journalism \& Mass Communication Quarterly, 92(1), 56-76. https://doi.org/10.1177\%2F1077699014559499

Kuz, A., Falco, M., Nahuel, L., y Giandini, R. S. (2015). Análisis de Redes Sociales a través de Gephi y NodeXL. Paper presented at the II Simposio Argentino Sobre Tecnología y Sociedad (STS)-JAIIO 44. https://bit.ly/2NWBzX2

Lee, J. K., Choi, J., Kim, C. y Kim, Y. (2014). Social media, network heterogeneity, and opinion polarization. Journal of Communication, 64(4), 702-722. https://doi.org/10.1111/jcom.12077

Matakos, A., Terzi, E., y Tsaparas, P. (2017). Measuring and moderating opinion polarization in social networks. Data Mining and Knowledge Discovery, 31(5), 14801505. https://doi.org/10.1007/s10618-017-0527-9

McSweeney, P. J. (2009). Gephi network statistics. Google Summer of Code, 1-8. https://bit.ly/3sk1qa5

Meneses, M., Martin-del-Campo, A. y Rueda-Zarate, H. (2018). \#TrumpenMexico. Transnational connective action on Twitter and the border wall dispute. Comunicar. Media Education Research Journal, 26(1). https://doi.org/10.3916/C55-2018-04

Papacharissi, Z. (2002). The virtual sphere: The internet as a public sphere. New Media \& Society, 4(1), 9-27.https://doi.org/10.1177\%2F14614440222226244

Quattrociocchi, W., Scala, A., y Sunstein, C. R. (2016). Echo chambers on Facebook. https://dx.doi.org/10.2139/ssrn.2795110

Rim, H., Lee, Y. y Yoo, S. (2020). Polarized public opinion responding to corporate social advocacy: Social network analysis of boycotters and advocators. Public Relations Review, 46(2), 101869. https://doi.org/10.1016/j.pubrev.2019.101869

Rodríguez, D. E. A., Aguilar, E. y Hung, E. S. (2010). Identidad y subjetividad en las redes sociales virtuales. Zona Próxima, (12) https://bit.ly/3qLqGpj

Rojas Hernández, H. y Puig-i-Abril, E. (2007). Comunicación y participación política Colombia 2006. Centro Para La Investigación En Comunicación Política, Facultad De Comunicación Social, Bogotá, Universidad Externado De Colombia y Centro Para La Comunicación y Democracia, University of Winsconsin Madison. 
Urcola Eceiza, E. y Elezgarai Ibañez, I. La polarización y echo chambers en el debate de la \#LeydeEutanasia en Twitter

Sindermann, C., Elhai, J. D., Moshagen, M., y Montag, C. (2020). Age, gender, personality, ideological attitudes and individual differences in a person's news spectrum: how many and who might be prone to "filter bubbles" and "echo chambers" online? Heliyon, 6(1). https://doi.org/10.1016/j.heliyon.2020.e03214

Stroud, N. J. (2010). Polarization and partisan selective exposure. Journal of Communication, 60(3), 556-576. https://doi.org/10.1111/j.14602466.2010.01497.x

Sunstein, C. R. (2003). República.com. Paidós Ibérica

Taber, C. S. y Lodge, M. (2006). Motivated skepticism in the evaluation of political beliefs. American Journal of Political Science, 50(3), 755-769. https://doi.org/10.1111/j.1540-5907.2006.00214.x

Törnberg, P. (2018). Echo chambers and viral misinformation: Modeling fake news as complex contagion. PloS One, 13(9). https://doi.org/10.1371/journal.pone.0203958

Tsai, W. S., Tao, W., Chuan, C., y Hong, C. (2020). Echo chambers and social mediators in public advocacy issue networks. Public Relations Review, 46(1). https://doi.org/10.1016/j.pubrev.2020.101882

UNESCO (Ed.) (-). Libertad de prensa en los medios. https://bit.ly/3soEpmD

Urman, A. (2020). Context matters: political polarization on Twitter from a comparative $\begin{array}{llll}\text { perspective. Media, Culture \& Society, 42(6), 857-879. } & \text { \& }\end{array}$ https://doi.org/10.1177\%2F0163443719876541

Vaccari, C., Valeriani, A., Barberá, P., Jost, J. T., Nagler, J. y Tucker, J. A. (2016). Of echo chambers and contrarian clubs: Exposure to political disagreement among German and Italian users of Twitter. Social Media Society, 2(3). https://doi.org/10.1177\%2F2056305116664221

Weber, I., Garimella, V. R. K. y Batayneh, A. (2013). Secular vs. islamist polarization in egypt on twitter. Paper presented at the Proceedings of the 2013 IEEE/ACM International Conference on Advances in Social Networks Analysis and Mining, 290297. https://doi.org/10.1145/2492517.2492557

Wu, A. X. (2013). Ideological polarization over a China-as-superpower mindset: An exploratory charting of belief systems among Chinese Internet users, 2008-2011. International Journal of Communication, 8, 2243-2272. https://bit.ly/2NzrX4A

Zuiderveen Borgesius, F., Trilling, D., Möller, J., Bodó, B., De Vreese, C. H. y Helberger, N. (2016). Should we worry about filter bubbles? Internet Policy Review. Journal on Internet Regulation, 5(1). https://bit.ly/37ISO4Y 


\section{AUTORES:}

\section{Eider Urcola Eceiza}

Doctoranda en el programa "Comunicación Social" del departamento de Comunicación Audiovisual y Publicidad en la Universidad del País Vasco/Euskal Herriko Unibertsitatea. Realiza el doctorado con una beca predoctoral otorgada por la Universidad del País Vasco/Euskal Herriko Unibertsitatea en el marco del Programa Predoctoral de Formación de Personal Investigador. Ha cursado estudios de Comunicación Audiovisual y un master en Psicología, todos ellos en la Universidad del País Vasco/Euskal Herriko Unibertsitatea. Ha trabajado en el departamento de comunicación de Udako Euskal Unibertsitatea.

eider.urcola@ehu.eus

Orcid ID: https://orcid.org/0000-0003-3199-2981

ResearchGate: https://www.researchgate.net/profile/Eider-Urcola-Eceiza

\section{Imanol Elezgarai Ibañez}

Doctorando del "Programa de doctorado en Sociedad, Política y Cultura" del departamento de Ciencia Política y de la Administración de la Universidad del País Vasco (EHU-UPV), en co-tutoría con el departamento de Periodismo. Realiza el doctorado con una beca predoctoral otorgada por el Gobierno Vasco en el marco del Programa Predoctoral de Formación de Personal Investigador No Doctor en la convocatoria del año 2019. Ha cursado los grados de Periodismo y el de Comunicación Audiovisual, así como el máster de Gobernanza y Estudios Políticos, todos ellos en la Universidad del País Vasco. Ha trabajado también varios años en diversos medios de comunicación vascos.

imanol.elezgaray@ehu.eus

Orcid ID: https://orcid.org/0000-0003-3067-0200 